\title{
Accounting for Social Risk Does not Eliminate Race/Ethnic Disparities in COVID-19 Infection Among Insured Adults: a Cohort Study
}

\author{
Jodi K. McCloskey, $M P H^{7}$, Jennifer L. Ellis, MBA, MSPH², Connie S. Uratsu, RN, MS, PHN ${ }^{7}$, \\ Melanie L. Drace, $M P H^{2}$, James D. Ralston, $M D, M P H^{3}$, Elizabeth A. Bayliss, MD, $M S P H^{2}$, \\ and Richard W. Grant, MD, MPH
}

'Division of Research, Kaiser Permanente Northern California, 2000 Broadway, Oakland, CA, USA; ${ }^{2}$ Institute for Health Research, Kaiser Permanente Colorado, Aurora, CO, USA; ${ }^{3}$ Kaiser Permanente Washington Health Research Institute, Kaiser Permanente Washington, Seattle, WA, USA.

BACKGROUND: Communities of color have been disproportionately impacted by the COVID-19 epidemic in the USA. OBJECTIVES: To examine the relationship of selfreported social health needs with SARS-COV-2 infection by race/ethnicity among insured adults with access to high-quality health care.

DESIGN AND PARTICIPANTS: A prospective cohort study of 26,741 adult Kaiser Permanente Northern California members insured by Medicaid and 58,802 Kaiser Permanente Colorado members insured by Medicare Advantage who completed social risk assessments prior to the onset of the COVID-19 pandemic.

MAIN MEASURES: We examined the independent relationships of demographic, medical, and social factors on SARS-COV-2 testing and positivity between March 1, 2020, and November 30, 2020, by race/ethnicity.

KEY RESULTS: Findings were similar in the two cohorts, with Latino (16-18\%), Asian (11-14\%), and Black (11-12\%) members having the highest prevalence of SARS-COV-2 infection (ORs adjusted for age, gender, and use of interpreter ranging from 1.68 to 2.23 compared to White member [7$8 \%], p<0.001)$. Further adjustment for medical comorbidity (e.g., obesity, diabetes, chronic lung disease); neighborhood measures; and self-reported social risk factors (e.g., trouble paying for basics, food insecurity, housing concerns, transportation barriers) did not appreciably change these results. CONCLUSIONS: Compared to non-Latino White members, members of other race/ethnic groups had higher positivity rates that were only minimally reduced after controlling for medical and neighborhood conditions and self-reported social risk factors. These findings suggest that traditional infection transmission factors such as essential work roles and household size that have disproportionate representation among communities of color may be important contributors to SARS-COV-2 infection among insured adults.

KEY WORDS: COVID-19; Socioeconomic factors; Disparity; Race; Ethnicity.

J Gen Intern Med 37(5):1183-90

DOI: $10.1007 / \mathrm{s} 11606-021-07261-y$

(c) Society of General Internal Medicine 2021

Received June 4, 2021

Accepted October 29, 2021

Published online February 2, 2022

\section{INTRODUCTION}

The COVID-19 epidemic has further exposed the chronic problem of race/ethnic health disparities in the USA, with some of the highest SARS-COV-2 infection and mortality rates within Latino and Black communities. ${ }^{1-3}$ Inadequate access to high-quality health care has been posited as a major factor driving health disparities, ${ }^{4-6}$ although more recent literature has focused on socially determined risk factors 7,8 and structural racism ${ }^{9-11}$ as important contributors to health disparities. Social risk factors (e.g., financial need, transportation barriers, food insecurity, social isolation, and/or mental stress) are disproportionally experienced by low-income communities of color and these factors are associated with poorer health outcomes. ${ }^{12}$ Structural racism is the term used to describe the underlying, long-standing societal factors that are a root cause of disproportionate social risks. ${ }^{13-15}$ Particularly relevant to the spread of SARS-COV-2 infection, Latino Black and Asian Americans are more likely to live in multigenerational housing. ${ }^{12}$ Latino and Black Americans are also more likely to work in lower-income service industry jobs that may not offer sick leave and have been deemed essential during the pandemic. ${ }^{13-15}$

Understanding contributing factors to race/ethnic disparities in SARS-COV-2 infection rates is an important step towards identifying potential solutions to reduce the impact of this epidemic and to prepare more effectively for the next one. We hypothesized that among adults with similar access to high-quality health care, social risk factors would help explain the association of race/ethnicity with COVID-19-related disparities. We therefore examined the association of SARSCOV-2 testing and positivity with race/ethnicity, socioeconomic status, and self-reported social factors among insured patients within two large, integrated care systems.

We investigated the extent to which observed disparities in SARS-COV-2 infection among non-White race/ethnic groups reflected differences in disease burden and comorbidities, neighborhood measures, and self-reported social risk factors. In examining the contribution of social risk, our intent was to improve understanding of how socially driven barriers to care contributed to health inequity during the pandemic so that 
health systems can better tailor care delivery to meet the current and future needs of these high-risk populations.

\section{METHODS Setting and Study Population}

We conducted this study within two integrated care delivery systems with membership representative of the larger communities they serve-Kaiser Permanente Northern California (KPNC) and Kaiser Permanente Colorado (KPCO). ${ }^{16}$ Eligible study members included adults aged 18 years or older on March 1, 2020, who completed a Medicaid new member survey within the past 2 years (KPNC) or an annual Medicare Health Risk Assessment survey (KPCO) within the past year. These member surveys are administered by health system staff and documented in the member's electronic health record captured member-identified health status, living situation, and social risk factors including food insecurity, financial strain, and housing concerns.

We measured SARS-COV-2 testing and positivity rates between March 1, 2020, and November 30, 2020. Member survey responses, demographics, diagnoses, and laboratory results were extracted directly from the KPNC and KPCO EPIC-based electronic medical record systems and from each site's Virtual Data Warehouse, a research database that aggregates member health data in a consistent, standardized format from multiple internal sources and externally billed claims. ${ }^{17}$

\section{Exposure and Covariates}

The primary exposure was self-reported race/ethnicity. Member race/ethnicity and need for interpreter services were captured at the time of health plan enrollment or by medical assistants and clerks during outpatient or inpatient care contacts. We determined chronic medical conditions using the ICD-10 diagnosis code list from the Centers for Medicare and Medicaid Chronic Conditions Warehouse, and we calculated the Elixhauser comorbidity score using 2 years of historical visit information. ${ }^{18}$ We defined members as obese based on body mass index $>30 \mathrm{~kg} / \mathrm{m}^{2}$ as calculated from most recent visit height and weight measurements. Social factors were coded from responses to member health assessment questions about worry related to food running out; eating fewer than two meals a day; trouble paying for basics such as food, housing, and utilities; concerns about housing safety, affordability, or transience; lack of transportation to make medical appointments or do activities of daily living (ADLs; e.g., getting out of bed or chair, bathing/showering/dressing/eating); and limitations on performing ADLs and instrumental ADLs (IADLs; e.g., managing medicines and finances, shopping for groceries). Responses also provided information about the presence of others in the household or if the member did not live independently.
The U.S. Census-based Social Vulnerability Index (SVI) score and component measures were obtained from the CDC and linked to member geocoded addresses. ${ }^{19}$ The SVI composite sum score ranges from 0 to 15 and incorporates measures of census tract demographics, socioeconomic status, and housing factors such as unemployment, poverty, and proportion of residents living in crowded housing.

\section{Outcomes}

Our primary outcomes were SARS-CoV-2 testing and infection between March 1 and November 30, 2020. We designated members as tested if their electronic medical records contained a completed SARS-CoV-2 PCR test or outpatient and inpatient visit with a COVID-19 screening ICD-10 diagnosis. Among the tested members, we classified those with a positive result for SARS-CoV-2 laboratory test or a confirmed COVID-19 diagnosis as positive for COVID-19 infection.

\section{Analytic Approach}

We used a staged modeling approach to describe differences in testing and positivity by race/ethnicity, and we examined the contribution of measured social risk factors to these differences. We assessed the baseline cohort characteristics on March 1, 2020.

We performed logistic regression on the dichotomous outcomes of having been tested (entire cohort) and of testing positive (among those who were tested). To obtain risk estimates, we fit a population-average model with generalized estimating equations and an independent correlation structure. This approach appropriately accounted for the within-cluster correlation in covariates measured at the census tract-level (Social Vulnerability Index score) among members residing within the same census tract and is robust to misspecification of the working correlation structure. ${ }^{20}$ Model 1 is adjusted for age, sex, and need for an interpreter. Model 2 represents added medical and mental health conditions that are risk factors for poor COVID-19 outcomes or are potential confounders due to their influence on the likelihood a medical provider would order testing and the members' ability to obtain testing. ${ }^{25}$ Conditions included diabetes, chronic obstructive pulmonary disease, asthma, hypertension, cardiovascular disease, hyperlipidemia, depression, anxiety, Alzheimer's disease, and related dementias, and the Elixhauser score for a measure of overall comorbidity burden. The final model, Model 3, represents added dichotomous self-reported social factors and the continuous neighborhood-level SVI score. Social factors in the KPNC models included living with others, children in household, education less than high school, trouble paying for basics, food insecurity, housing concerns, and help needed with ADLs. Social factors in the KPCO models included education less than high school, not living independently, food insecurity, and limitations in ADLs and limitations in instrumental ADLs. ${ }^{26,27}$ All descriptive and statistical analyses were performed using SAS 9.4. Our research was approved by the 
Kaiser Permanente Institutional Review Board and all procedures followed were in accordance with the ethical standards of the IRB and the Helsinki Declaration of 1975, as revised in 2000 .

\section{RESULTS}

\section{Cohort Characteristics}

We studied 26,741 adult KPNC members insured by Medicaid in Northern California and 58,802 KPCO members in Colorado covered by Medicare Advantage who completed a member health assessment and had membership on March 1, 2020. As expected, given differences in program eligibility criteria, the Northern California Medicaid cohort was younger (age $42.7 \pm 17.0$ vs. $73.6 \pm 6.6$ years, $p<0.001)$ and more diverse ( $68.8 \%$ vs. $17.4 \%$ non-White, $p<0.001$ ) than the Colorado Medicare cohort (Tables 1 and 2).

Nearly two-thirds (65\%) of the Northern California cohort were women. Latino and Black members tended to live in neighborhoods with higher social vulnerability than Asian and White members. Latino members were most likely to live in areas with a larger proportion of crowded housing and to report having children in their household (49\%) and less than a high school education (20\%). Black members reported the highest prevalence of financial need (34\%), food insecurity (28\%), housing concerns $(23 \%)$, and transportation barriers (19\%) (Table 1).

The majority (55.9\%) of the Colorado Medicare cohort were women. As in the Northern California cohort, Latino and Asian members were most likely to report less than a high school education (20\% and 16\%). Black, Latino, and Asian members were more likely to report food insecurity (22\%, $20 \%$, and $18 \%$ ) than other groups. Black members were the most likely to have difficulty with ADLs and IADLs (13\% and 17\%) (Table 2).

\section{COVID-19 Testing and Positivity}

In the Northern California Medicaid cohort, 7548 members (28\%) were tested and of these 933 tested positive (12\%) for SARS-COV-2 infection. The overall testing and positivity rates were lower in the Colorado Medicare cohort, with 8,253 members tested (14\%) and of these 684 tested positive $(8 \%)$. Testing and positivity rates varied significantly by race/ethnicity (Figure 1).

Table 1 Northern California-Baseline Characteristics of Patients Completing the Medicaid Onboarding Survey by Race and Ethnicity

\begin{tabular}{|c|c|c|c|c|c|c|c|}
\hline & $\begin{array}{l}\text { All } \\
\text { patients }\end{array}$ & White & $\begin{array}{l}\text { Latino/ } \\
\text { Hispanic }\end{array}$ & Asian & Black & $\begin{array}{l}\text { Multi-racial/ } \\
\text { other }\end{array}$ & Unknown \\
\hline Patients, $n$ ( $\%$ of cohort) & 26,741 & $\begin{array}{l}8,344 \\
(31.2)\end{array}$ & $6,827(25.5)$ & $\begin{array}{l}5,181 \\
(19.4)\end{array}$ & $\begin{array}{l}4,014 \\
(15.0)\end{array}$ & $1,250(4.7)$ & $1,125(4.2)$ \\
\hline Age in years, mean (SD) & $42.7(17.0)$ & $43.1(16.2)$ & $40.0(16.6)$ & $47.2(18.5)$ & $40.9(15.8)$ & $43.9(18.3)$ & $42.1(17.0)$ \\
\hline Women, \% & 65.0 & 61.6 & 69.0 & 62.8 & 70.0 & 67.5 & 54.5 \\
\hline \multicolumn{8}{|l|}{ Insurance type*, \% } \\
\hline Medicaid & 74.9 & 73.8 & 76.8 & 71.2 & 78.2 & 71.2 & 79.6 \\
\hline Dual-eligible & 12.8 & 12.6 & 10.8 & 15.5 & 12.6 & 17.5 & 8.0 \\
\hline Commercial & 10.1 & 10.8 & 10.6 & 10.8 & 7.3 & 9.3 & 10.7 \\
\hline Medicare/other & 2.3 & 2.8 & 1.7 & 2.5 & 1.9 & 2.0 & 1.7 \\
\hline \multicolumn{8}{|l|}{ Diagnoses, \% } \\
\hline Diabetes & 15.2 & 12.1 & 15.7 & 18.7 & 15.2 & 19.8 & 14.7 \\
\hline COPD/asthma & 20.0 & 21.7 & 19.1 & 13.0 & 28.1 & 6.7 & 9.8 \\
\hline Anxiety & 24.8 & 33.0 & 25.5 & 12.4 & 24.6 & 27.2 & 14.1 \\
\hline Obesity (BMI 30+) & 40.5 & 41.3 & 48.7 & 20.4 & 53.8 & 41.6 & 28.9 \\
\hline Depression & $\begin{array}{l}6,159 \\
(23.5)\end{array}$ & $\begin{array}{l}2,485 \\
(30.3)\end{array}$ & $1,533(22.8)$ & $599(11.8)$ & $\begin{array}{l}1,094 \\
(27.7)\end{array}$ & $322(26.0)$ & $126(11.9)$ \\
\hline Elixhauser score, mean (SD) & $2.2(2.4)$ & $2.3(2.5)$ & $2.1(2.3)$ & $1.8(2.1)$ & $2.6(2.6)$ & $2.6(2.6)$ & $1.2(1.6)$ \\
\hline $\begin{array}{l}\text { Any primary care visits in prior year, } \\
\%\end{array}$ & 67.7 & 66.2 & 68.7 & 69.9 & 66.4 & 69.0 & 65.0 \\
\hline Any hospitalizations in prior year, \% & 10.2 & 9.9 & 11.5 & 8.5 & 12.7 & 10.1 & 4.4 \\
\hline Any ED visits in prior year, \% & 35.4 & 35.5 & 37.5 & 23.5 & 49.8 & 40.7 & 21.2 \\
\hline Needs an interpreter, $\%$ & 8.5 & 2.1 & 16.3 & 15.9 & 0.3 & 4.2 & 10.0 \\
\hline $\begin{array}{l}\text { Social Vulnerability Index score, mean } \\
\text { (SD) }\end{array}$ & $7.43(2.16)$ & $6.80(2.04)$ & $7.99(2.10)$ & $7.20(2.16)$ & $8.18(2.04)$ & $7.39(2.16)$ & $7.21(2.15)$ \\
\hline$\%$ crowded housing, mean (SD) & $6.7(5.6)$ & $5.0(4.6)$ & $8.3(6.1)$ & $6.7(5.7)$ & $7.7(5.5)$ & $6.4(5.4)$ & $6.4(5.7)$ \\
\hline \multicolumn{8}{|l|}{ Self-reported social context and risk, } \\
\hline Lives with others & 81.6 & 78.1 & 85.3 & 87.0 & 74.3 & 82.0 & 85.5 \\
\hline Children in the household & 40.7 & 33.7 & 49.0 & 38.1 & 44.5 & 37.1 & 45.2 \\
\hline Education less than high school & 12.4 & 7.0 & 20.4 & 15.5 & 7.5 & 9.3 & 11.0 \\
\hline Trouble paying for basics & 22.2 & 22.4 & 20.8 & 15.2 & 34.3 & 23.8 & 15.3 \\
\hline Food insecurity & 18.8 & 17.1 & 19.9 & 14.1 & 27.5 & 20.7 & 12.7 \\
\hline Housing concerns & 14.5 & 13.4 & 13.1 & 11.7 & 23.0 & 17.4 & 11.3 \\
\hline Transportation barriers & 10.9 & 10.0 & 9.9 & 8.0 & 19.0 & 12.3 & 6.5 \\
\hline Needs help with ADLs & 15.4 & 15.3 & 13.2 & 12.6 & 22.0 & 22.6 & 9.4 \\
\hline
\end{tabular}

All p-values < 0.01; SD standard deviation, COPD chronic obstructive pulmonary disease, BMI body mass index, ED emergency department, $A D L$ activities of daily living

*Members initially enrolled in Medicaid could subsequently change insurance type over the subsequent year 
Table 2 Colorado-Baseline Characteristics of Patients Completing the Medicare Total Health Assessment Within the Past 2 Years by Race/Ethnicity

\begin{tabular}{|c|c|c|c|c|c|c|c|}
\hline & $\begin{array}{l}\text { All } \\
\text { patients }\end{array}$ & White & $\begin{array}{l}\text { Latino/ } \\
\text { Hispanic }\end{array}$ & Asian & Black & $\begin{array}{l}\text { Multi-racial/ } \\
\text { other }\end{array}$ & Unknown \\
\hline Patients, $n$ (\% of cohort) & 58,802 & $\begin{array}{l}48,587 \\
(82.6 \%)\end{array}$ & $5,097(8.7 \%)$ & $\begin{array}{l}938 \\
(1.6 \%)\end{array}$ & $\begin{array}{l}1,450 \\
(2.5 \%)\end{array}$ & $1,542(2.6 \%)$ & $\begin{array}{l}1,188 \\
(2.0 \%)\end{array}$ \\
\hline Age in years, mean (SD) & $73.6(6.6)$ & $73.8(6.6)$ & $72.5(6.6)$ & $73.1(6.5)$ & $73.6(6.5)$ & $73.3(6.4)$ & $73.0(6.6)$ \\
\hline Women, \% & 55.9 & 54.6 & 60.2 & 57.4 & 56.3 & 49.7 & 55.8 \\
\hline \multicolumn{8}{|l|}{ Diagnoses, \% } \\
\hline Diabetes & 21.3 & 18.9 & 37.7 & 30.9 & 39.9 & 23.7 & 16.6 \\
\hline COPD/asthma & 23.7 & 23.9 & 21.8 & 17.1 & 27.7 & 25.4 & 20.2 \\
\hline Hypertension & 57.1 & 56.1 & 62.7 & 55.3 & 80.7 & 57.8 & 46.3 \\
\hline Cardiovascular disease & 33.2 & 33.7 & 30.9 & 24.6 & 33.4 & 33.5 & 27.0 \\
\hline Obesity (BMI 30+) & 31.2 & 30.8 & 37.6 & 10.2 & 39.9 & 30.3 & 27.2 \\
\hline Hyperlipidemia & 76.5 & 76.4 & 79.0 & 77.6 & 79.9 & 76.5 & 66.2 \\
\hline Anxiety/PTSD & 14.1 & 14.2 & 15.4 & 7.8 & 11.6 & 14.5 & 10.5 \\
\hline Depression & 18.5 & 19.0 & 18.0 & 8.5 & 15.0 & 15.7 & 13.6 \\
\hline Alzheimer's/dementia & 4.9 & 4.6 & 6.8 & 4.1 & 7.5 & 5.2 & 3.6 \\
\hline Elixhauser score, mean (SD) & $3.1(2.5)$ & $3.0(2.5)$ & $3.4(2.6)$ & $2.5(2.2)$ & $3.8(2.6)$ & $3.01(2.5)$ & $2.5(2.4)$ \\
\hline $\begin{array}{l}\text { Any primary care visits in prior } 2 \text { years, } \\
\%\end{array}$ & 99.3 & 99.4 & 99.4 & 99.7 & 99.7 & 99.3 & 94.2 \\
\hline Any hospitalizations in prior 2 years, $\%$ & 15.0 & 15.3 & 14 & 8.8 & 15.4 & 14.6 & 13.5 \\
\hline Any ED visits in prior 2 years, $\%$ & 23.7 & 23.6 & 25.5 & 18.4 & 26.1 & 24.6 & 20.5 \\
\hline Needs an interpreter, \% & 0.3 & 0.0 & 2.1 & 5.5 & 0.0 & 0.5 & 0.1 \\
\hline $\begin{array}{l}\text { Social Vulnerability Index (SVI) score, } \\
\text { mean (SD) }\end{array}$ & $6.4(2.2)$ & $6.3(2.1)$ & $7.6(2.4)$ & $6.4(2.2)$ & $7.8(2.4)$ & $6.6(2.3)$ & $6.5(2.2)$ \\
\hline$\%$ crowded housing, mean (SD) & $2.0(2.8)$ & $1.8(2.5)$ & $3.3(3.9)$ & $2.0(2.9)$ & $3.8(4.2)$ & $2.1(2.8)$ & $1.9(2.6)$ \\
\hline \multicolumn{8}{|l|}{ Self-reported social context and risk, \% } \\
\hline Do not live independently & 2.0 & 2.1 & 1.3 & 1.4 & 1.4 & 1.5 & 1.7 \\
\hline Education less than high school & 4.9 & 3.0 & 19.7 & 15.6 & 6.1 & 6.1 & 4.1 \\
\hline Food insecurity & 10.2 & 8.6 & 19.6 & 17.8 & 22.3 & 12.6 & 11.6 \\
\hline ADL limitation & 7.9 & 7.6 & 9.5 & 7.6 & 12.9 & 9.3 & 7.3 \\
\hline Instrumental ADL limitation & 11 & 10.5 & 13.9 & 10.9 & 17 & 11.3 & 8.8 \\
\hline
\end{tabular}

All p-values < 0.01; SD standard deviation, COPD chronic obstructive pulmonary disease, BMI body mass index, ED emergency department, ADL activities of daily living

We adjusted these results using our staged modeling approach (Tables 3 and 4). The observed univariate relationship of a greater likelihood of testing and positivity remained among Latino members across all adjusted models in both cohorts (Medicaid cohort: RR 1.60, CI 1.32-1.94; Medicare cohort: RR 1.81, CI 1.48-2.22) compared to the White referent group controlling for age, sex, chronic conditions, socioeconomic and household, and self-reported social risks. Asian and Black Medicare members were also at elevated risk for infection (RR 1.90, CI $1.10,3.28$, and RR 1.46 CI 1.04, 2.05). Inclusion of selfreported social risk factors to our fully adjusted model did not significantly attenuate these disparities.

In the Medicaid cohort, additional factors associated with a higher risk of SARS-COV-2 infection included younger age, children in the household, and social vulnerability (Appendix). In the Medicare cohort, additional factors associated with higher risk of COVID-19 infection included having less than a high school education, social vulnerability, requiring help from others for daily living, and diagnoses of COPD, obesity, or Alzheimer's disease (Appendix).

\section{DISCUSSION}

In this cohort of insured adults, self-reported race/ethnicity was the strongest contributor to differences in SARS-COV-
2 testing and infection after controlling for age, gender, language barriers, underlying health conditions, and census tract household- and neighborhood-level measures. The novel contribution of this analysis is that additional adjustment for self-reported social risk factors did not materially change these associations. After additional adjustment for self-reported social risks, we found that Latino members from both our Medicaid and Medicare populations had a significantly higher risk of infection despite having similar access to care. Among older adults insured by Medicare, we found that Black and Asian members were also at elevated risk of contracting SARS-COV-2 infection compared to White members.

Race/ethnic COVID-19 disparities have been reported in other US contexts. For example, a nationwide study involving over 5 million mostly male (91\%) individuals receiving care via the US Department of Veterans Affairs also found double the rates of SARS-COV-2 infection among non-White compared to White recipients even after adjusting for demographic and medical comorbidity differences. ${ }^{21}$ This study was not able to measure and account for social risk. Another study by Vahidy et al. using medical and census-level data from a large medical center in Houston found that living in high population density neighborhoods at least partially mediated the relationship between non-White race/ethnicity and SARS-CoV-2 infection. ${ }^{22}$ This analysis also lacked individual respondent selfreported social risk factors. 


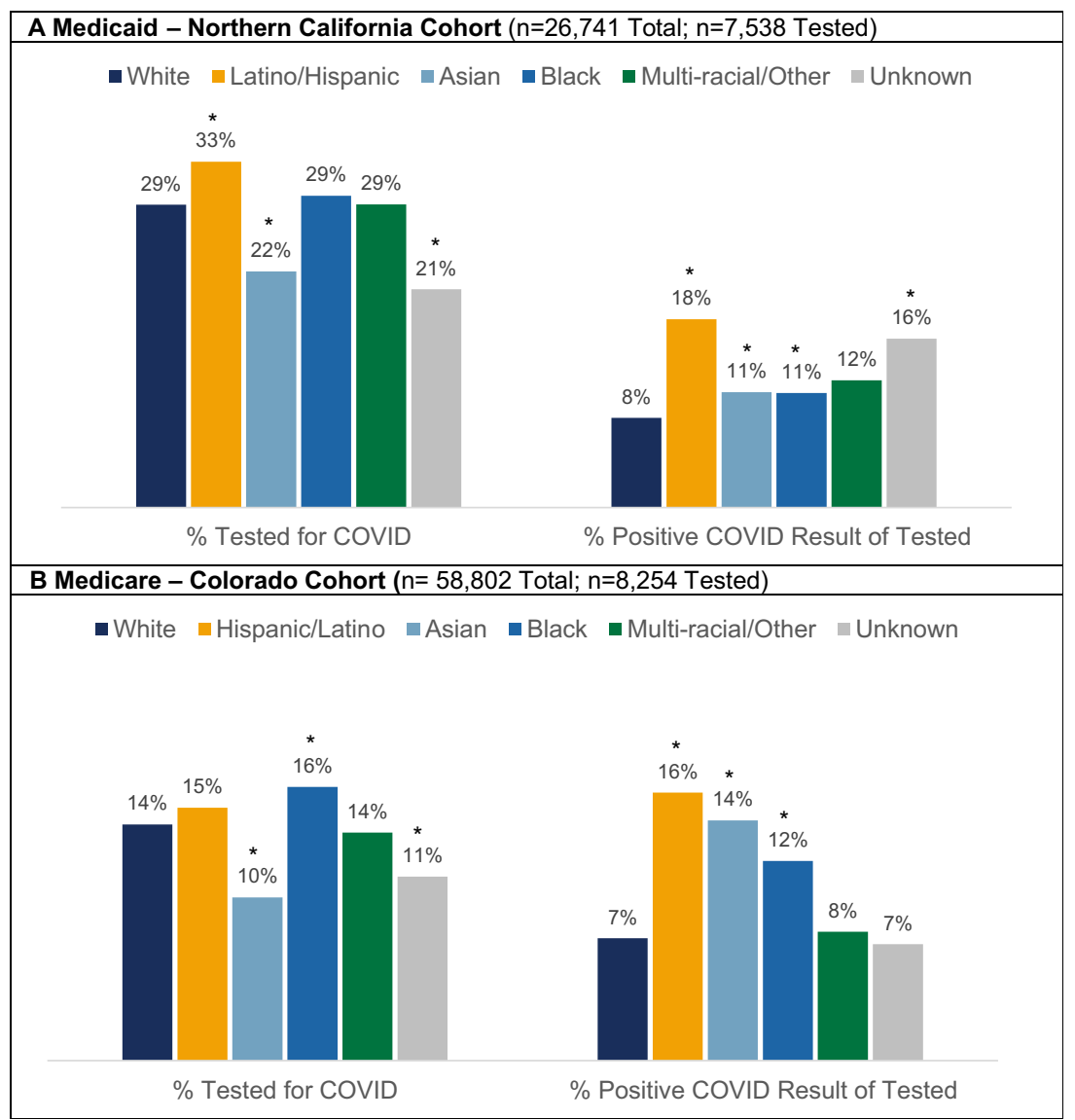

Figure 1. COVID-19 testing and positivity rates by race/ethnicity. A Medicaid-Northern California Cohort ( $n=26,741$ Total; $n=7,538$ Tested). B Medicare - Colorado Cohort $(n=58,802$ Total; $n=8,254$ Tested). Testing for COVID-19 includes patients with a diagnostic COVID-19 lab result or an encounter with a COVID-19 screening or lab confirmed COVID-19 diagnosis. "“*” indicates unadjusted $p$-value $<0.05$ in comparison to the White referent group.

Table 3 Likelihood of COVID-19 Testing and Positivity by Race/Ethnicity. Medicaid—Northern California Cohort

\begin{tabular}{|c|c|c|c|c|c|c|c|c|c|c|c|c|}
\hline \multicolumn{2}{|c|}{$\begin{array}{l}\text { (A) Tested for COVID- } \\
19\end{array}$} & \multirow[b]{2}{*}{$\begin{array}{l}p- \\
\text { value }\end{array}$} & \multirow[b]{2}{*}{$\begin{array}{l}\text { Model } 2 \\
\text { RR } \\
\text { (95\% CI) }\end{array}$} & \multirow[b]{2}{*}{$\begin{array}{l}p \text { - } \\
\text { value }\end{array}$} & \multirow[b]{2}{*}{$\begin{array}{l}\text { Model } 3 \\
\text { RR } \\
\text { (95\% CI) }\end{array}$} & \multirow[b]{2}{*}{$\begin{array}{l}p \text { - } \\
\text { value }\end{array}$} & \multicolumn{3}{|c|}{$\begin{array}{l}\text { (B) Positive COVID-19 Test } \\
\text { Result }\end{array}$} & \multirow[b]{2}{*}{$\begin{array}{l}p \text { - } \\
\text { value }\end{array}$} & \multirow[b]{2}{*}{$\begin{array}{l}\text { Model } 3 \\
\text { RR } \\
\text { (95\% CI) }\end{array}$} & \multirow[b]{2}{*}{$\begin{array}{l}p \text { - } \\
\text { value }\end{array}$} \\
\hline Covariate & $\begin{array}{l}\text { Model 1 } \\
\text { RR } \\
\text { (95\% CI) }\end{array}$ & & & & & & $\begin{array}{l}\text { Model 1 } \\
\text { RR } \\
\text { (95\% CI) }\end{array}$ & $\begin{array}{l}p \text { - } \\
\text { value }\end{array}$ & $\begin{array}{l}\text { Model } 2 \\
\text { RR } \\
\text { (95\% CI) }\end{array}$ & & & \\
\hline \multicolumn{13}{|l|}{$\begin{array}{l}\text { Race/ } \\
\text { ethnicity }\end{array}$} \\
\hline $\begin{array}{l}\text { White, } \\
\text { non- } \\
\text { Hispanic }\end{array}$ & $\begin{array}{l}1.00 \\
\text { (referent) }\end{array}$ & & $\begin{array}{l}1.00 \\
\text { (referent) }\end{array}$ & & $\begin{array}{l}1.00 \\
\text { (referent) }\end{array}$ & & $\begin{array}{l}1.00 \\
\text { (referent) }\end{array}$ & & $\begin{array}{l}1.00 \\
\text { (referent) }\end{array}$ & & $\begin{array}{l}1.00 \\
\text { (referent) }\end{array}$ & \\
\hline $\begin{array}{r}\text { Latino/ } \\
\text { Hispanic }\end{array}$ & $\begin{array}{l}1.17 \\
(1.11 \\
1.22)\end{array}$ & $<0.01$ & $\begin{array}{l}1.20 \\
(1.14, \\
1.26)\end{array}$ & $<0.01$ & $\begin{array}{l}1.19 \\
(1.13 \\
1.26)\end{array}$ & $<0.01$ & $\begin{array}{l}1.84 \\
(1.55 \\
2.20)\end{array}$ & $<0.01$ & $\begin{array}{l}1.71 \\
(1.43 \\
2.04)\end{array}$ & $<0.01$ & $\begin{array}{l}1.60 \\
(1.32, \\
1.94)\end{array}$ & $<0.01$ \\
\hline Black & $\begin{array}{l}1.01 \\
(0.95 \\
1.07)\end{array}$ & 0.69 & $\begin{array}{l}0.99 \\
(0.94, \\
1.05)\end{array}$ & 0.83 & $\begin{array}{l}0.98 \\
(0.92 \\
1.04)\end{array}$ & 0.49 & $\begin{array}{l}1.26 \\
(1.03 \\
1.55)\end{array}$ & 0.03 & $\begin{array}{l}1.22 \\
(0.99 \\
1.51)\end{array}$ & 0.06 & $\begin{array}{l}1.22 \\
(0.97 \\
1.52)\end{array}$ & 0.08 \\
\hline Asian & $\begin{array}{l}0.80 \\
(0.75 \\
0.85)\end{array}$ & $<0.01$ & $\begin{array}{l}0.92 \\
(0.86, \\
0.98)\end{array}$ & 0.01 & $\begin{array}{l}0.91 \\
(0.84 \\
0.97)\end{array}$ & 0.01 & $\begin{array}{l}1.21 \\
(0.98 \\
1.49)\end{array}$ & 0.07 & $\begin{array}{l}1.07 \\
(0.87, \\
1.33)\end{array}$ & 0.52 & $\begin{array}{l}1.06 \\
(0.84, \\
1.34)\end{array}$ & 0.63 \\
\hline $\begin{array}{l}\text { Multi- } \\
\text { racial/other }\end{array}$ & $\begin{array}{l}1.00 \\
(0.91, \\
1.09)\end{array}$ & 0.99 & $\begin{array}{l}1.00 \\
(0.91, \\
1.09)\end{array}$ & 0.94 & $\begin{array}{l}1.00 \\
(0.91, \\
1.10)\end{array}$ & 0.96 & $\begin{array}{l}1.40 \\
(1.01 \\
1.94)\end{array}$ & 0.04 & $\begin{array}{l}1.36 \\
(0.99 \\
1.88)\end{array}$ & 0.06 & $\begin{array}{l}1.17 \\
(0.81, \\
1.70)\end{array}$ & 0.40 \\
\hline Unknown & $\begin{array}{l}0.75 \\
(0.66 \\
0.85)\end{array}$ & $<0.01$ & $\begin{array}{l}0.88 \\
(0.78, \\
1.00)\end{array}$ & 0.05 & $\begin{array}{l}0.89 \\
(0.78, \\
1.02)\end{array}$ & 0.09 & $\begin{array}{l}1.76 \\
(1.26 \\
2.46)\end{array}$ & 0.00 & $\begin{array}{l}1.57 \\
(1.11 \\
2.20)\end{array}$ & 0.01 & $\begin{array}{l}1.52 \\
(1.08 \\
2.15)\end{array}$ & 0.02 \\
\hline
\end{tabular}

Model 1 controls for age, gender, and needs interpreter; Model 2 adds chronic medical diagnoses (obesity, diabetes, COPD, asthma, hypertension, hyperlipidemia, cardiovascular disease, depression, anxiety); Model 3 adds self-reported context and needs (lives with others, children in household, education less than high school, trouble paying for basics, food insecurity, housing concerns, needs help with ADLs, transportation barriers) and Social Vulnerability Index Score. 
Table 4 Likelihood of COVID-19 Testing and Positivity by Race/Ethnicity. Medicare—Colorado Cohort

\begin{tabular}{|c|c|c|c|c|c|c|c|c|c|c|c|c|}
\hline \multicolumn{2}{|c|}{$\begin{array}{l}\text { (A) Tested for COVID- } \\
19\end{array}$} & \multirow[b]{2}{*}{$\begin{array}{l}p- \\
\text { value }\end{array}$} & \multirow[b]{2}{*}{$\begin{array}{l}\text { Model } 2 \\
\text { RR } \\
\text { (95\% CI) }\end{array}$} & \multirow[b]{2}{*}{$\begin{array}{l}p \text { - } \\
\text { value }\end{array}$} & \multirow[b]{2}{*}{$\begin{array}{l}\text { Model } 3 \\
\text { RR } \\
\text { (95\% CI) }\end{array}$} & \multirow[b]{2}{*}{$\begin{array}{l}p \text { - } \\
\text { value }\end{array}$} & \multicolumn{3}{|c|}{$\begin{array}{l}\text { (B) Positive COVID-19 test } \\
\text { result }\end{array}$} & \multirow[b]{2}{*}{$\begin{array}{l}p \text { - } \\
\text { value }\end{array}$} & \multirow[b]{2}{*}{$\begin{array}{l}\text { Model } 3 \\
\text { RR } \\
\text { (95\% CI) }\end{array}$} & \multirow[b]{2}{*}{$\begin{array}{l}p \text { - } \\
\text { value }\end{array}$} \\
\hline Covariate & $\begin{array}{l}\text { Model 1 } \\
\text { RR } \\
\text { (95\% CI) }\end{array}$ & & & & & & $\begin{array}{l}\text { Model 1 } \\
\text { RR } \\
\text { (95\% CI) }\end{array}$ & $\begin{array}{l}p- \\
\text { value }\end{array}$ & $\begin{array}{l}\text { Model 2 } \\
\text { RR } \\
\text { (95\% CI) }\end{array}$ & & & \\
\hline \multicolumn{13}{|l|}{$\begin{array}{l}\text { Race/ } \\
\text { ethnicity }\end{array}$} \\
\hline $\begin{array}{l}\text { White, } \\
\text { Non- } \\
\text { Hispanic }\end{array}$ & $\begin{array}{l}1.00 \\
\text { (referent) }\end{array}$ & & $\begin{array}{l}1.00 \\
\text { (referent) }\end{array}$ & & $\begin{array}{l}1.00 \\
\text { (referent) }\end{array}$ & & $\begin{array}{l}1.00 \\
\text { (referent) }\end{array}$ & & $\begin{array}{l}1.00 \\
\text { (referent) }\end{array}$ & & $\begin{array}{l}1.00 \\
\text { (referent) }\end{array}$ & \\
\hline $\begin{array}{r}\text { Latino/ } \\
\text { Hispanic }\end{array}$ & $\begin{array}{l}1.06 \\
(0.98 \\
1.14)\end{array}$ & 0.13 & $\begin{array}{l}1.07 \\
(1.00, \\
1.15)\end{array}$ & 0.05 & $\begin{array}{l}1.08 \\
(1.00, \\
1.16)\end{array}$ & 0.05 & $\begin{array}{l}2.23 \\
(1.84, \\
2.71)\end{array}$ & $<0.01$ & $\begin{array}{l}1.86 \\
(1.52, \\
2.28)\end{array}$ & $<0.01$ & $\begin{array}{l}1.81 \\
(1.48, \\
2.22)\end{array}$ & $<0.01$ \\
\hline Black & $\begin{array}{l}1.15 \\
(1.02 \\
1.29)\end{array}$ & 0.03 & $\begin{array}{l}1.14 \\
(1.01, \\
1.28)\end{array}$ & 0.04 & $\begin{array}{l}1.14 \\
(1.01, \\
1.28)\end{array}$ & 0.04 & $\begin{array}{l}1.68 \\
(1.21 \\
2.32)\end{array}$ & $<0.01$ & $\begin{array}{l}1.49 \\
(1.07 \\
2.06)\end{array}$ & 0.02 & $\begin{array}{l}1.46 \\
(1.04, \\
2.05)\end{array}$ & 0.03 \\
\hline Asian & $\begin{array}{l}0.69 \\
(0.56 \\
0.84)\end{array}$ & $<0.01$ & $\begin{array}{l}0.78 \\
(0.64 \\
0.95)\end{array}$ & 0.01 & $\begin{array}{l}0.77 \\
(0.64 \\
0.94)\end{array}$ & 0.01 & $\begin{array}{l}1.97 \\
(1.15, \\
3.37)\end{array}$ & 0.01 & $\begin{array}{l}1.91 \\
(1.11, \\
3.30)\end{array}$ & 0.02 & $\begin{array}{l}1.90 \\
(1.10, \\
3.28)\end{array}$ & 0.02 \\
\hline $\begin{array}{l}\text { Multi- } \\
\text { racial/other }\end{array}$ & $\begin{array}{l}0.96 \\
(0.85 \\
1.09)\end{array}$ & 0.56 & $\begin{array}{l}0.97 \\
(0.86 \\
1.10)\end{array}$ & 0.65 & $\begin{array}{l}0.96 \\
(0.85 \\
1.09)\end{array}$ & 0.57 & $\begin{array}{l}1.07 \\
(0.66, \\
1.72)\end{array}$ & 0.79 & $\begin{array}{l}1.03 \\
(0.65, \\
1.65)\end{array}$ & 0.89 & $\begin{array}{l}1.05 \\
(0.66 \\
1.67)\end{array}$ & 0.84 \\
\hline Unknown & $\begin{array}{l}0.78 \\
(0.66 \\
0.92)\end{array}$ & $<0.01$ & $\begin{array}{l}0.83 \\
(0.70, \\
0.97)\end{array}$ & 0.02 & $\begin{array}{l}0.83 \\
(0.70, \\
0.97)\end{array}$ & 0.02 & $\begin{array}{l}0.98 \\
(0.52 \\
1.83)\end{array}$ & 0.95 & $\begin{array}{l}1.01 \\
(0.54, \\
1.88)\end{array}$ & 0.98 & $\begin{array}{l}0.99 \\
(0.53 \\
1.86)\end{array}$ & 0.98 \\
\hline
\end{tabular}

Model 1 controls for age, gender, and needs interpreter; Model 2 adds chronic medical diagnoses (obesity, diabetes, COPD, asthma, hypertension, cardiovascular disease, hyperlipidemia, depression, anxiety, Alzheimer's/dementia, serious mental illness); Model 3 adds self-reported context and needs (living independently, education less than high school, food insecurity, assistance needed with activities of daily living (ADLs) and instrument $A D L s)$ and Social Vulnerability Index Score

Social risk factors such as food insecurity, housing concerns, transportation barriers, and financial needs have been identified as potent contributors to race/ethnic health disparities, particularly related to chronic diseases such as diabetes and heart disease. ${ }^{23-25}$ Even among the insured population studied here, risk factors such as food insecurity and trouble paying for basics were prevalent (ranging from 10 to $22 \%$ overall) and much more common among non-White race/ethnic groups. The main insight from our analyses is that controlling for these race/ethnic social factor differences did not appreciably diminish the significantly higher rates of SARS-COV-2 infection among Latinos (or older Black and Asian Medicare recipients). These findings give further weight to the impact of traditional air-borne infection risk factors, namely living and working in close proximity to others.

Prior studies have documented that lower-income working Americans were more likely to have service-related jobs deemed "essential" during the COVID-19 pandemic. ${ }^{26,27}$ These workplaces increase risk of infectious exposure, whereas most individuals who were able to "work from home" during the pandemic were able to substantially reduce their exposure risk. Among those aged 65 and older, Latino, Black, and Asian Americans were more likely to remain in the workforce ${ }^{28,29}$ and work in low-wage essential jobs than White Americans. Non-White Americans were also more likely to live in larger, multigenerational households. ${ }^{30}$ Thus, our results provide further support that inability to socially distance ${ }^{31}$ may be the primary causal factor underlying race/ethnic SARS-CoV-2 transmission disparities. ${ }^{32}$
Our results must be interpreted within the context of our study design. Although this is one of the largest studies to incorporate self-reported social risks, these factors were assessed up to 1-2 years prior to the onset of the COVID-19 pandemic and may potentially have changed during the course of the pandemic. In addition, we were unable to directly measure individual level exposure to traditional air-borne infection risk factors, leaving us to infer that living situation and workplace setting may explain persistent race/ethnic disparities based on census tract data. Finally, our analyses were limited to Medicaid-insured individuals in California and Medicare-insured individuals in Colorado and may not be generalizable to other regions of the country.

Our study provides further evidence of the impact of structural racism on the health of US race/ethnic minority communities during the COVID-19 pandemic. ${ }^{33}$ We found that even among individuals with equal access to high-quality insurance, there were marked race/ethnic differences in becoming infected with SARS-COV-2. These differences persisted after accounting for age, gender, and medical comorbidity. The lack of attenuation of infectious risk after further accounting for both neighborhood-level measures and individual, selfreported social risk factors suggest that while social conditions can play a significant role in health, our current US experience of COVID-19 may be driven by structural factors more specifically related to infection transmission such as crowded housing coupled with "essential" in-person employment during a period of rapid viral spread. These insights should inform equitable health protection efforts, namely increasing COVID- 
19 vaccination rates ${ }^{34}$; addressing unemployment benefit legislation, equitable housing, and workforce policy reform; and other policy measures to contain the impact of the ongoing COVID pandemic and in preparation for the next infectious disease epidemic.

Acknowledgements: This project was supported by Kaiser Permanente's Garfield Memorial Fund.

Corresponding Author: Jodi K. McCloskey, MPH; Division of Research, Kaiser Permanente Northern California, 2000 Broadway, Oakland, CA 94612, USA (e-mail: jodi.k.mccloskey@kp.org).

Electronic supplementary material The online version contains supplementary material available at https://doi.org/10.1007/ s11606-021-07261-y.

\section{Declarations:}

Conflict of Interest: The authors declare that they do not have a conflict of interest.

\section{REFERENCES}

1. Gold JAW, Rossen LM, Ahmad FB, Sutton P, Li Z, Salvatore PP, et al. Race, Ethnicity, and Age Trends in Persons Who Died from COVID-19 United States, May-August 2020. MMWR Morb Mortal Wkly Rep. 2020;69(42):1517-21

2. Romano SD, Blackstock AJ, Taylor EV, El Burai Felix S, Adjei S, Singleton CM, et al. Trends in Racial and Ethnic Disparities in COVID19 Hospitalizations, by Region - United States, March-December 2020. MMWR Morb Mortal Wkly Rep. 2021:70(15):560-5.

3. Rossen LM, Branum AM, Ahmad FB, Sutton P, Anderson RN. Excess Deaths Associated with COVID-19, by Age and Race and Ethnicity United States, January 26-October 3, 2020. MMWR Morb Mortal Wkly Rep. 2020;69(42):1522-7.

4. Ayanian JZ, Weissman JS, Schneider EC, Ginsburg JA, Zaslavsky AM. Unmet health needs of uninsured adults in the United States. Jama. 2000;284(16):2061-9.

5. Call KT, McAlpine DD, Garcia CM, Shippee N, Beebe T, Adeniyi TC, et al. Barriers to care in an ethnically diverse publicly insured population: is health care reform enough? Med Care. 2014;52(8):720-7.

6. Hadley J. Sicker and poorer-the consequences of being uninsured: a review of the research on the relationship between health insurance, medical care use, health, work, and income. Med Care Res Rev. 2003;60(2 Suppl):3S-75S; discussion 6S-112S.

7. Healthy People 2030. [Internet]: U.S. Department of Health and Human Services, Office of Disease Prevention and Health Promotion; [2021 May 20]; Social Determinants of Health]. Available from: https://health. gov/healthypeople/objectives-and-data/social-determinants-health. Accessed 20 May 2021

8. Chetty R, Stepner M, Abraham S, Lin S, Scuderi B, Turner N, et al. The Association Between Income and Life Expectancy in the United States, 2001-2014. JAMA. 2016;315(16):1750-66.

9. Bailey ZD, Feldman JM, Bassett MT. How structural racism works racist policies as a root cause of U.S. Racial health inequities. N Engl J Med. 2021 Feb 25;384(8):768-73.

10. Bailey ZD, Krieger N, Agénor M, Graves J, Linos N, Bassett MT. Structural racism and health inequities in the USA: evidence and interventions. Lancet. 2017 Apr 8;389(10077):1453-63.

11. Glance LG, Thirukumaran CP, Dick AW. The Unequal Burden of COVID 19 Deaths in Counties With High Proportions of Black and Hispanic Residents. Med Care. 2021 Jun 1;59(6):470-6.

12. Cohn Dv, Passel JS. A record 64 million Americans live in multigenerational households. Pew Research Center [Internet]. 2018. Available from: https://www.pewresearch.org/fact-tank/2018/04/05/a-record-64- million-americans-live-in-multigenerational-households/ft_18-03-27_ multigeneration_trend/. Accessed 5 Apr 2021.

13. Bureau of Labor Statistics UDoL. Hispanics and Latinos in industries and occupations. The Economics Daily [Internet]. 2015 July 21, 2020. Available from: https://www.bls.gov/opub/ted/2015/hispanics-and-latinos-in-industriesand-occupations.htm. Accessed 20 May 2021.

14. Bureau of Labor Statistics UDoL. Household Data Annual Averages Table 10. Employed persons by occupation, race, Hispanic or Latino ethnicity, and sex. Labor Force Statistics from Current Population Survey 2021. Available from: https://www.bls.gov/cps/cpsaat10.htm. Accessed 20 May 2021.

15. Legislatures NCoS. COVID-19: Essential Workers in the States. 2021 [updated January 11, 2021 October 12, 2020]; Available from: https:// www.ncsl.org/research/labor-and-employment/covid-19-essentialworkers-in-the-states.aspx\#Map. Accessed 20 May 2021

16 16.Gordon N, Lin T. The Kaiser Permanente Northern California Adult Member Health Survey. Perm J. 2016 Fall;20(4):15-225.

17 Ross TR, Ng D, Brown JS, Pardee R, Hornbrook MC, Hart G, et al. The HMO Research Network Virtual Data Warehouse: A Public Data Model to Support Collaboration. EGEMS (Wash DC). 2014 Mar 24;2(1):1049.

18. Elixhauser A, Steiner C, Harris DR, Coffey RM. Comorbidity measures for use with administrative data. Med Care. 1998 Jan;36(1):8-27.

19. Flanagan BE, Hallisey EJ, Adams E, Lavery A. Measuring Community Vulnerability to Natural and Anthropogenic Hazards: The Centers for Disease Control and Prevention's Social Vulnerability Index. J Environ Health. 2018;80(10):34-6.

20. Liang K-Y, Zeger SL. Longitudinal data analysis using generalized linear models. Biometrika. 1986;73(1):13-22.

21. Rentsch CT, Kidwai-Khan F, Tate JP, Park LS, King JT, Jr., Skanderson $\mathrm{M}$, et al. Patterns of COVID-19 testing and mortality by race and ethnicity among United States veterans: A nationwide cohort study. PLoS Med. 2020;17(9):e1003379.

22. Vahidy FS, Nicolas JC, Meeks JR, Khan O, Pan A, Jones SL, et al. Racial and ethnic disparities in SARS-CoV-2 pandemic: analysis of a COVID-19 observational registry for a diverse US metropolitan population. BMJ Open. 2020;10(8):e039849.

23. Berkowitz SA, Hulberg AC, Standish S, Reznor G, Atlas SJ. Addressing Unmet Basic Resource Needs as Part of Chronic Cardiometabolic Disease Management. JAMA Internal Medicine. 2017;177(2):244-52.

24. Berkowitz SA, Meigs JB, DeWalt D, Seligman HK, Barnard LS, Bright OJM, et al. Material Need Insecurities, Control of Diabetes Mellitus, and Use of Health Care Resources: Results of the Measuring Economic Insecurity in Diabetes Study. JAMA Internal Medicine. 2015;175(2):257-65.

25. Knighton AJ, Stephenson B, Savitz LA. Measuring the effect of social determinants on patient outcomes: a systematic literature review. Journal of health care for the poor and underserved. 2018;29(1):81-106.

26. Bureau of Labor Statistics UDoL. Job Flexibilities and Work Schedules 2017-2018: Data from the American Time Use Survey. US Bureau of Labor Statistics. 2019. Available from: https://www.bls.gov/news.release/flex2.htm. Accessed 20 May 2021.

27. Bureau of Labor Statistics UDoL. Labor force characteristics by race and ethnicity, 2019: Report 1088. US Bureau of Labor Statistics. 2020. Bureau of Labor Statistics UDoL. Labor force characteristics by race and ethnicity, 2019: Report 1088. US Bureau of Labor Statistics. 2020. Available from: https://www.bls.gov/opub/reports/ race-andethnicity/2019/home.htm. Accessed 19 Apr 2021.

28. Bureau of Labor Statistics UDoL. Household Data Annual Averages Table 3. Employment status of the civilian noninstitutional population by age, sex, and race. Labor Force Statistics from Current Population Survey 2021. Available from: https://www.bls.gov/cps/cpsaat03.htm. Accessed 20 May 2021.

29. Bureau of Labor Statistics UDoL. Household Data Annual Averages Table 4. Employment status of the Hispanic or Latino population by age and sex. Labor Force Statistics from Current Population Survey 2021. Available from: https://www.bls.gov/cps/cpsaat04.htm. Accessed 20 May 2021.

30. Schramm JF, Carlos. The U.S. Essential Workforce Ages 50 and Older. AARP Public Policy Institute. 2020 September; Fact Sheet 655. Available from: https://doi.org/10.26419/ppi.00111.001.

31. Paul LA, Daneman N, Brown KA, Johnson J, van Ingen T, Joh E, et al Characteristics associated with household transmission of SARS-CoV-2 in Ontario, Canada: A cohort study. Clinical Infectious Diseases. 2021;ciab186. https://doi.org/10.1093/cid/ciab186.

32 Rodriguez-Diaz CE, Guilamo-Ramos V, Mena L, Hall E, Honermann B, Crowley JS, et al. Risk for COVID-19 infection and death among Latinos in 
the United States: examining heterogeneity in transmission dynamics. Annals of Epidemiology. 2020;52:46-53.e2.

33. Bailey ZD, Krieger N, Agénor M, Graves J, Linos N, Bassett MT. Structural racism and health inequities in the USA: evidence and interventions. The Lancet. 2017;389(10077): 1453-63.

34. Bibbins-Domingo K, Petersen M, Havlir D, editors. Taking vaccine to where the virus is - equity and effectiveness in coronavirus vaccinations.
JAMA Health Forum; 2021;2(2):e210213. https://doi.org/10.1001/ jamahealthforum.2021.0213.

Publisher's Note: Springer Nature remains neutral with regard to jurisdictional claims in published maps and institutional affiliations. 